\title{
A Survey on Performance Analysis of Different Transforms in DWT for Image Compression
}

\author{
Sowmyashree M S ${ }^{1}$, Vivek $\mathrm{M}^{2}$, Rahul $\mathrm{R}^{3}$,Sukruth B $\mathrm{S}^{4}$ \\ Department of Telecommunication Engineering, BMSIT ${ }^{1,2,3,4}$ \\ Assistant Professor ${ }^{l}$
}

\begin{abstract}
The use of different digital devices is increasing every day. Thus it is necessary to compress images to make efficient transfer and storage of data. Wavelet is the image compression algorithm. It is Discrete Wavelet Transforms (DWT). Wavelet algorithms can process data at different scales or resolutions. The different wavelets used for image compression are Haar, Daubechies, Biorthogonal and Symlet. This paper presents a comparative study of various wavelet families for image compression and tests the results in terms of compressed image quality. The results are measured in terms of MSE, PSNR, CR and Elapsed time for compression considering as subjective quality measures.
\end{abstract}

Index Terms: Wavelet, Haar, Symlet, Daubechies, Biorthogonal, MSE, CR,PSNR, Elapsed time

\section{INTRODUCTION}

Image compression is defined as the process of reducing the amount of data required to represent digital image. An image contains large information, which brings in difficulties for storage, processing and transmission. Thus image compression is very important and necessary. There are two ways of image compression (1) lossless compression and (2) lossy compression. Lossy image compression algorithms are applicable whenever the exact reconstruction of an image is not required. In recent years, efforts have been made to design image compression methods in which the main goal is to obtain good quality of decompressed images even at very low bit rates.

A digital image is mainly composed by edges, edge associated details and textures and it is very necessary to preserve this information to get a good quality of reconstructed image. The Discrete Cosine Transform was used for information coding in order to save the parts of a natural image.

The ISO/CCITT Joint Photographic Experts Group (JPEG) selected the DCT for its baseline coding technique [2]. The fidelity loss in JPEG coding occurs entirely in quantization. However, the quantization tables are image-dependent and the complexity of the encoder is rather high.

Hence JPEG-2000 has selected the Wavelet Transform (WT) for its baseline coding technique [3]. Wavelets provide good compression ratios, especially for high resolution images.

Recently, Discrete Wavelet Transform (DWT) has emerged as a popular Technique for image compression applications with excellent compression performance. The WT requires much less hardware to implement than Fourier methods, such as the DCT. One of the shortcomings of using DCT is blocking artifact and bad subjective quality when the images are restored by this method at the high compression ratios. Therefore, much of the research activities in image compression have been focused on the discrete wavelet transform. WT of an image represents image as a sum of wavelets on multi-resolution levels. In wavelet transform any onedimensional function is transformed into a two-dimensional space, where it is approximated by coefficients that depend on time and on scale. Hence the wavelet transform is well suited for image compression. The type of wavelet transform will directly impact on of the complexity of the calculation and, indirectly, affect the image compression and reconstruct the image with an acceptable error.

\section{Discrete Wavelet transform}

The DWT is based on sub-band coding, is found to yield a fast computation of Wavelet Transforms. It is easy to implement and reduce the computation time and required resources. DWT adopt fast algorithm of two dimensional wavelet transform. The original image is decomposed into four sub-bands after passing a high- pass filter and low- pass filter [4]. The combination of high pass $\mathrm{g}[\mathrm{n}]$ and low pass filter $\mathrm{h}[\mathrm{n}]$ comprise a pair of analyzing filters. The output of each filter contains half the frequency content, but an equal amount of samples as the input signal.

The four sub-bands are LL, HL, LH and HH respectively. LL is a low frequency sub-band of the approximate image. HL is a high frequency sub-band of the horizontal details of the image. LH is a high frequency sub-band of the vertical details of the image. HH is a high frequency sub-band of the diagonal details of the image. The process is called the first level of wavelet decomposition. The low frequency sub-band can be continually decomposed into four sub-bands. 


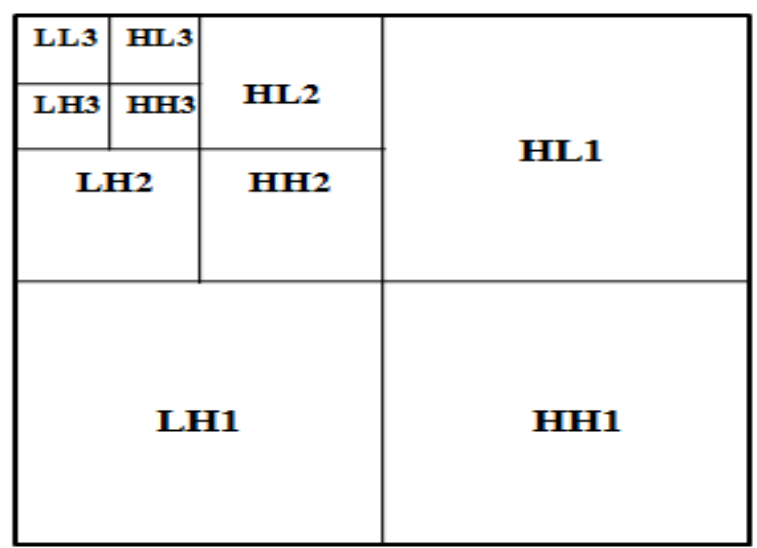

Level of Wavelet decomposition

\section{Various Wavelet Used For Image Compression}

This paper has used four wavelets: Haar, Daubechies, Symlet and Biorthogonal wavelet for image compression.

\section{Haar Transform}

Haar wavelet is the first and simplest [5].Haar wavelet is discontinuous and resembles a step function. The attracting features of the Haar transform, including fast implementation and able to analyze the local feature, make it a potential candidate in modern electrical and computer engineering applications, such as signal and image compression.

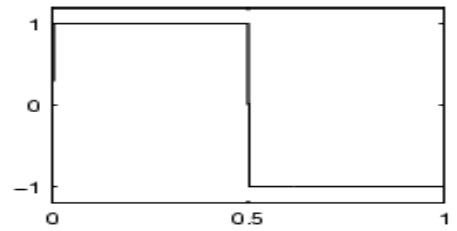

Wavelet function psi

Haar Wavelet Function Waveform

\section{Daubechies Transform}

It is first wavelet family of scale functions that are orthogonal and have finite vanishing moments that is compact support. The wavelet is near symmetric.

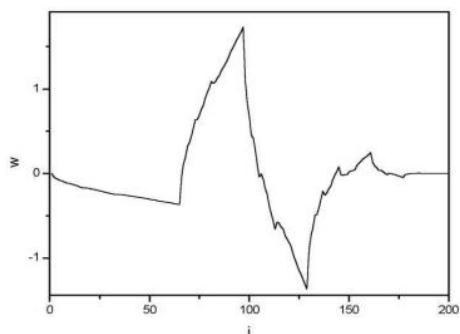

Daubechies Wavelet Function Waveform

\section{Symlet Transform}

The Symlets are symmetrical wavelets proposed by Daubechies as modifications to the db family. The properties of the two wavelet families are very similar. There are 7 types of Symlets functions and they are from sym2 to sym8. We have used sym2 function below. 


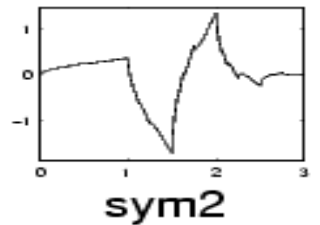

Symlet Wavelet Function Waveform

\section{Biorthogonal Transform}

This family of wavelets exhibits the property of linear phase needed for signal and reconstruction of image. Using two wavelets i.e, one for decomposition (on the left side) and the other for reconstruction (on the right side) rather than the same single one, interesting properties are derived.

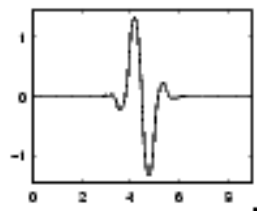

\section{bior1.5}

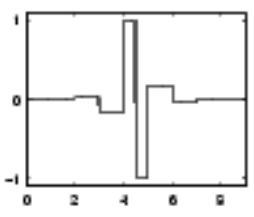

Biorthogonal Wavelet Function Waveform

\section{AlgorithM For IMAge COMPRESSION USING DWT}

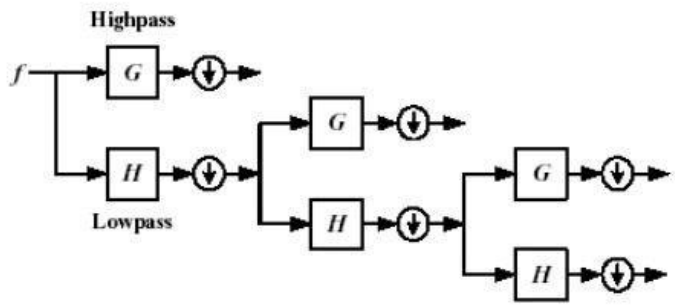

Multilevel Decomposition using low pass and high pass filters for image compression using wavelets

Algorithm follows a quantization approach that divides the input image in 4 filter coefficients and then performs further quantization on the lower order filter. This quantization depends upon the decomposition levels and maximum numbers of decomposition levels to be entered are 3 for DWT. DWT exploits interpixel redundancies to render excellent decorrelation for most natural images. Thus the uncorrelated transform coefficients can be encoded independently without compromising coding efficiency. DWT packs energy in the low frequency regions.

Therefore, some of the high frequency content can be discarded without significant quality degradation. Such a quantization scheme causes further reduction in the entropy or average number of bits per pixel.

The attributes of the DWT have led to its widespread deployment in virtually every image/video processing standard of the last decade, for example, JPEG (classical), MPEG- 1, MPEG-2, MPEG-4, and MPEG-4 FGS. Nevertheless, the DWT still offers new research directions that are being explored in the current and upcoming image/video coding standards.

\section{Performance Criterion}

The performance is evaluated by the following parameters:

\section{Distortion measure}

Mean Square Error (MSE) is used to measure the rate of distortion in the reconstructed image. Mean square error (MSE) is widely used to measure the degree of image distortion because they can represent the overall gray-value error contained in the entire image and are mathematically tractable as well.

$$
M S E=\frac{1}{m n} \sum_{i=0}^{m-1} \sum_{j=0}^{n-1}[I(i, j)-K(i, j)]^{2}
$$




\section{PSNR}

PSNR is used as an approximation to human perception of reconstruction quality. PSNR has been accepted as a widely used quality measurement in the field of image compression.

\section{Compression Ratio}

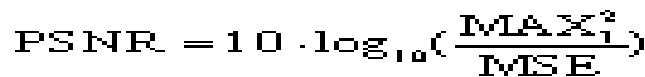

$$
\begin{aligned}
& =20 \cdot \log :\left(\frac{\Gamma \mathrm{MLS}}{\sqrt{\mathrm{TME}})}\right.
\end{aligned}
$$

The Compression ratio (CR) is the ratio between the size of the original image and the size of the compressed image.

$$
\text { Compression ratio }=\frac{N_{1}}{N_{2}}
$$

\section{Elapsed time for compression}

Time taken to compress the image from its original form to compressed form is known as Elapsed time for Compression.

\section{Simulation And Results}

DWT based image compression is performed to get the desired results. Input image is to be compressed to a certain level using DWT. Qualitative analysis have been performed by obtaining the compressed version of the input image by DWT Technique and comparing it with the input image. Quantitative analysis has been presented by measuring the values of attained Peak Signal to Noise Ratio and Compression Ratio at different decomposition levels. Uses of Haar wavelet, Daubechies wavelet, Symlets wavelet and Biorthogonal Wavelet have been done and a comparative analysis is carried out.

\section{LEVEL OF COMPRESSION}

\begin{tabular}{|c|c|c|c|c|}
\cline { 2 - 5 } \multicolumn{1}{c|}{} & Parameters & \multicolumn{2}{c|}{} \\
\hline Transform & MSE & PSNR & CR & $\begin{array}{c}\text { Elapsed time for } \\
\text { compression (s) }\end{array}$ \\
\hline Haar & 14.8433 & 36.4155 & 58.1999 & 3.6560 \\
\hline Daubechies & 12.1902 & 37.2707 & 55.6749 & 3.1250 \\
\hline Symlet & 11.7764 & 37.4207 & 57.4467 & 2.7500 \\
\hline Biorthogonal & 11.3684 & 37.5738 & 57.1085 & 2.4840 \\
\hline
\end{tabular}

II LEVEL OF COMPRESSION

\begin{tabular}{|c|c|c|c|c|}
\cline { 2 - 5 } \multicolumn{1}{c|}{} & \multicolumn{3}{c|}{ Parameters } \\
\cline { 2 - 5 } \multicolumn{1}{c|}{} & \multicolumn{3}{c|}{} \\
\hline Transform & MSE & PSNR & CR & $\begin{array}{c}\text { Elapsed time for } \\
\text { compression (s) }\end{array}$ \\
\hline Haar & 25.3072 & 34.0984 & 75.8566 & 2.5620 \\
\hline Daubechies & 24.7402 & 34.1968 & 72.7118 & 2.2398 \\
\hline Symlet & 22.598 & 34.5901 & 74.7086 & 2.0630 \\
\hline Biorthogonal & 21.3608 & 74.8346 & 74.5726 & 3.7500 \\
\hline
\end{tabular}

VI. Conclusion And Future Scope

DWT is considered as a faster approach since:

- The basic principle is to factorize the polyphase matrix of a wavelet filter into a sequence of alternating upper and lower triangular matrices and a diagonal matrix.

- This leads to the wavelet implementation by means of banded-matrix multiplications

DWT exploits interpixel redundancies to render excellent decorrelation for most natural images. Thus uncorrelated transform coefficients can be encoded independently without compromising coding efficiency. The DWT packs energy in the low frequency regions. Therefore the high frequency content can be discarded without significant quality degradation. Such a quantization scheme causes further reduction in the entropy or average number of bits per pixel. Peak signal to noise ratio (PSNR) as the objective quality measure. Hence we can say 
that the "best wavelet" choice of wavelet in the image compression of images is dependent on the image content and desired image quality.

- By analysing the first level image compression table we find that other transforms are better than Haar transforms since all parameters tend to show that Haar is less efficient.

- Compression ratio is better for Haar transform in both level of compressions as shown in the respective tables.

\section{References}

[1] R. C. Gonzalez and R. E. Woods, "Digital Image Processing”, Reading. MA: Addison Wesley, 2004

[2] V.Ratnakar and M.Livny. RD-OPT: An efficient algorithm for optimizing DCT quantization tables. Data Compression Conference.

[3] A.Skodras, Christopoulos and T Ebrahimi. The JPEG 2000 still image compression standard. IEEE Signal processing magazine, pp

[4] Sonja Grgic, Kresimir Kers, Mislav Grgic, "Image Compression Using Wavelets" IEEE, ISIE"e99Bled Slovenia.

[5] L.W.Chang, CY Wang and SM Lee Designing JPEG quantization tables based on Human Visual System. Signal Processing: Image Communication, Volume 16, Issue 5,501-506, Jan 2001.

[6] A. Islam and W.A. Pearlman, An embedded and efficient low-complexity hierarchical image coder, in Proc. SPIE Visual Comm. and Image Processing, vol. 3653, pp. 294-305, 1999.

[7] B. Kim and W.A. Pearlman, An embedded wavelet video coder using three-dimensional set partitioning in hierarchical tree, IEEE Data Compression Conference, pp.251-260, March 1997.

[8] Kumar,V., V.Sunil., Reddy,M.Indra Sena., "Image Compression Techniques by using Wavelet Transform ", Journal of information engineering and applications, Vol 2, No.5, 2012

[9] Katharotiya,Anilkumar., Patel,Swati., "Comparative Analysis between DCT \& DWT Techniques of Image Compression", Journal of information engineering and applications, Vol 1, No.2, 2011

[10] S. Mallat, Multifrequency channel decompositions of images and wavelet models, IEEE Trans. Acoust., Speech, Signal Processing, vol. 37, pp.2091-2110, Dec. 1989.

[11] A.N. Netravali and B.G. Haskell, Digital pictures, representation and compression, in Image Processing, Proc. Of Data Compression Conference, pp.252-260, 1997.

[12] E. Ordentlich, M. Weinberger, and G. Seroussi, A low-complexity modeling approach for embedded coding of wavelet coef_cients, in Proc. IEEE Data Compression Conf., Snowbird, UT, pp. 408-417, Mar. 1998.

[13] W.A. Pearlman, Performance bounds for subband codes, Chapter 1 in Subband Image Coding, J. W. Woods and Ed. Klvwer. Academic Publishers, 1991.

[14] Proposal of the arithmetic coder for JPEG2000, ISO/IEC/JTC1/SC29/WG1 N762, Mar. 1998.

[15] A. Said and W.A. Pearlman, A new, fast and ef_cient image codec based on set partitioning in hierarchical trees, IEEE Trans. on Circuits and Systems for Video Technology 6, pp. 243-250, June 1996. 